\title{
Photovoltaic Effect in Poly(vinylidene fluoride)
}

\author{
Hiroyuki Sasabe, Tsutomu NAKayama, Kinya KumaZAwa, \\ Seizo MIYATA, and Eiichi FUKADA* \\ Faculty of Technology, Tokyo University of Agriculture \& \\ Technology, Koganei, Tokyo 184, Japan. \\ *The Institute of Physical and Chemical Research, \\ Wako, Saitama 351, Japan.
}

(Received May 15, 1981)

\begin{abstract}
The generation of photocarriers and their transport processes in poled poly(vinylidene fluoride) films under light illumination are discussed from the viewpoint of a ferroelectric property of $\beta$ form crystals. We observed not only a pyroelectric current but an additional current due to anomalous photovoltaic effect. The open circuit photovoltage was estimated as $4 \times 10^{4} \mathrm{~V}$, which exceeds the band gap potential of PVDF more than three decades. Because of the high resistivity of PVDF, the conversion efficiency of light to electric energy is still quite low ( $c a .0 .25 \%$ ), but further improvements are suggested.

KEY WORDS Poly(vinylidene fluoride) / Ferroelectric Polymer Photovoltaic Effect / Pyroelectricity / Photocarrier Generation / Photoelectric Current /
\end{abstract}

Industrial applications of poly(vinylidene fluoride) (PVDF) have been extensive in recent years, resulting in remarkable product, such as electroacoustic transducers used in audio-speakers and microphones, ${ }^{1}$ ultrasonic diagnostic equipment in the medical electronics, ${ }^{2}$ ultrasonic hydrophones for the ocean technology, ${ }^{3}$ and the photo-electric (or pyro-electric) transducers ${ }^{4}$ for vidicon and IR sensors. These applications are based essentially on the ferroelectric property of PVDF, and its success in industrial application has made PVDF the most promising polymer as an "active material." Here we use the term "ferroelectric" for a polymer which undergoes a spontaneous (remanence) polarization and obeys Curie-Weiss' rule. A detailed morphological study of ferroelectric PVDF has also been carried out, ${ }^{5}$ and it was clarified that the ferroelectricity of PVDF is associated with the crystal structure of the $\beta$ form prepared, for example, by drawing or poling of the film.

According to the works of Fridkin ${ }^{6}$ and Glass $e t$ $a l .,^{7}$ the anomalous photovoltaic effect has been observed in inorganic ferroelectric crystals such as $\mathrm{BaTiO}_{3}$ and $\mathrm{LiNbO}_{3}$, in which open circuit photovoltage exceeds the band gap potential of the crys- tals by several orders of magnitude. This effect seems applicable for the conversion of solar energy into electric power. In this paper, we investigate the possibility of anomalous photovoltaic effect in a poled PVDF film, and discuss the generation of photocarriers and their transport in relation to photoconductive properties of PVDF.

\section{EXPERIMENTAL}

Under light excitation, electronic carriers (electrons or holes) are generated in a polymer film. If an electric field is applied across a sample, a current, observed in the external circuit, corresponds to a photocurrent $I_{\mathrm{ph}}$, whereas if an electric field is absent, then the current corresponds to a shortcircuit photovoltaic current $I_{\mathrm{pv}}$. We carried out measurements of $I_{\mathrm{ph}}$ and $I_{\mathrm{pv}}$ on both unpoled and poled PVDF samples as functions of temperature $(T)$ and wavelength $(\lambda)$ of the exciting light in the ranges of $20-120^{\circ} \mathrm{C}$ and $300-700 \mathrm{~nm}$. In the case of an $I_{\mathrm{ph}}$ measurement, the applied voltage across the sample ranged from 0 to $\pm 600 \mathrm{~V}$. Figure 1 shows a schematic diagram of the measuring system used in this study. A film sample (S) with semi- 


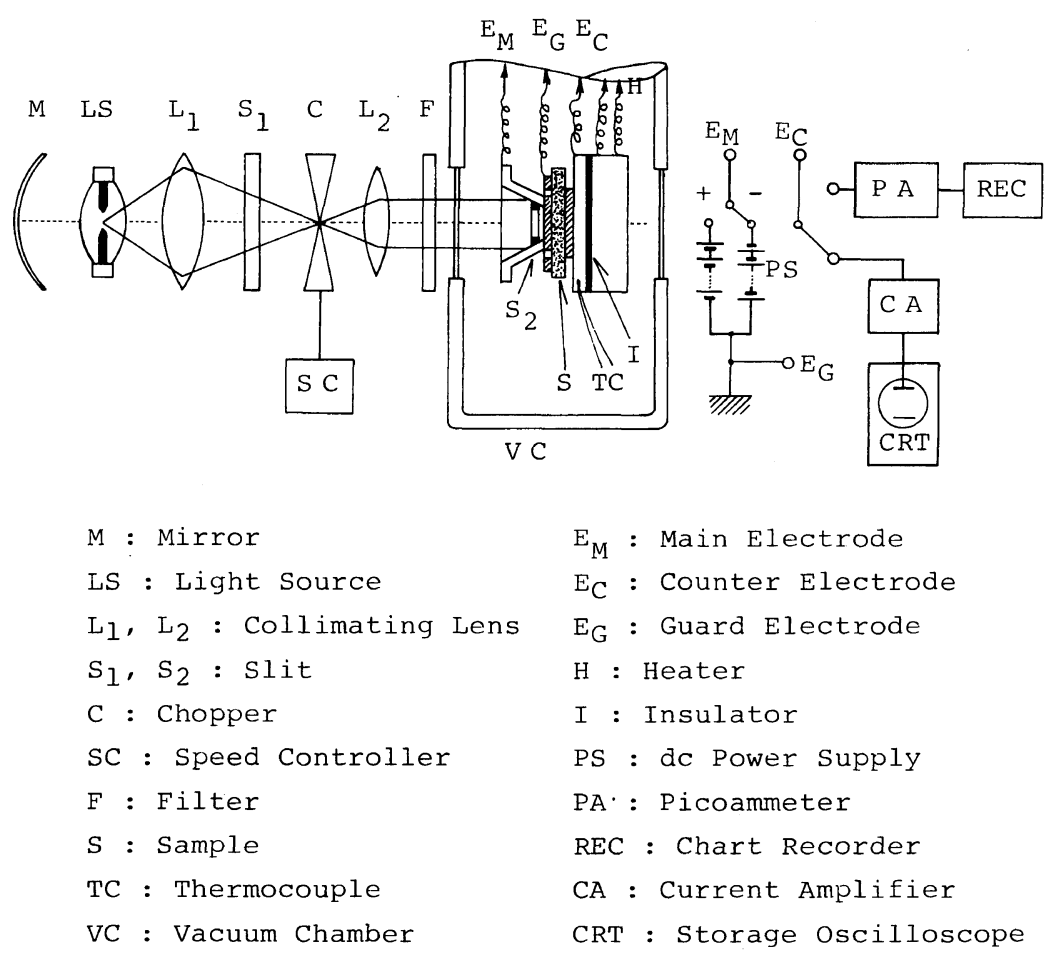

Figure 1. Schematic diagram of photocurrent measurements.

transparent gold electrodes is set in a vacuum chamber (VC), and illuminated with a steady source of (or chopped) light. This light source (LS) was a Xe lamp, monochromated with interference filters (F) or a monochromator (NIKON G250). The light intensity at each wavelength was calibrated with a pyroelectric radiant flux meter (MOLECTRON PR200). Chopped light obtained by a mechanical chopper (C) was available for the pulsed response of $I_{\mathrm{ph}}$ or $I_{\mathrm{pv}}$. The photocurrent $I_{\mathrm{ph}}$ (or the photovoltaic current $I_{\mathrm{pv}}$ ) was amplified with a high speed current amplifier (KEITHLEY 427) and recorded on a storage oscilloscope (HITACHI VC-830 or TEKTRONIX 7623A) in the case of fast responses and on a chart recorder (YOKOGAWA MODEL 3056) in the case of slow responses.

Three types of samples were used: an unpoled PVDF film $\left(S_{\mathrm{A}}\right)$ (KUREHA KF polymer with an $\alpha$ form crystal structure, $100 \mu \mathrm{m}$ thick), a dye-doped $S_{\mathrm{A}}$ film $\left(S_{\mathrm{B}}\right)$, and a poled PVDF film $\left(S_{\mathrm{C}}\right)$ (KUREHA KF piezo-film, $30 \mu \mathrm{m}$ thick). As for Sample $S_{\mathrm{B}}$, the acrydine-orange dye was doped in order to shift the optical absorption edge of $S_{\mathrm{A}}$ $(<170 \mathrm{~nm})$ to the visible wavelength region and enhance the quantum efficiency in this region. As electrodes, Au was semitransparently deposited ( $c a$. 300 A thick) in vacuo on both sides of each film.

\section{RESULTS AND DISCUSSION}

\section{Photocarrier Generation}

First, in order to check the possibility of photocarrier generation in the PVDF samples, the photocurrent measurement was carried out. With the unpoled sample $S_{\mathrm{B}}$, photocurrent $I_{\mathrm{ph}}$ increased gradually with time under step-wise illumination, as shown in Figure 2(a). This is a typical photocurrent response observed in most polymers with carrier traps and no space charge effect. Since the dark current was by one or two orders of magnitude smaller than the photocurrent (Figure 3), the excess carriers were generated by the light irradiation.

Figure 4 indicates the wavelength dependence of $I_{\mathrm{ph}}$ in $S_{\mathrm{A}} \cdot I_{\mathrm{ph}}$ is almost constant in the range of 400 $600 \mathrm{~nm}$. This parallels the change in transmittance of PVDF which is also constant (ca. 90\%) in this region (Figure 5). The excitation light is partly absorbed by the semitransparent Au electrode and 


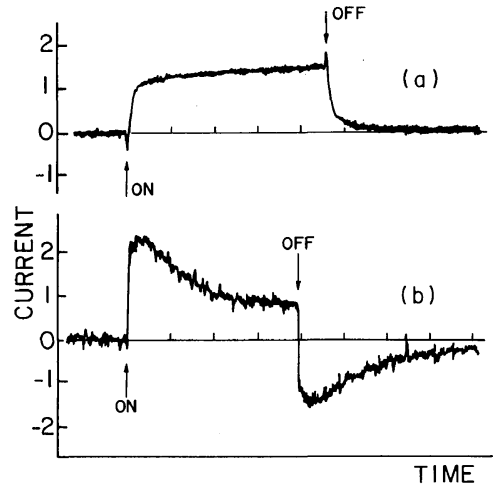

Figure 2. Photocurrent response in (a) the unpoled sample $S_{\mathrm{B}}$ and (b) the poled sample $S_{\mathrm{C}}$ under step-wise illumination. Scales are $8 \times 10^{-11} \mathrm{~A} / \mathrm{div}$ and $2 \mathrm{~s} / \mathrm{div}$, and the applied voltage $V_{\mathrm{a}}$ is $V_{\mathrm{a}}=270 \mathrm{~V}$.

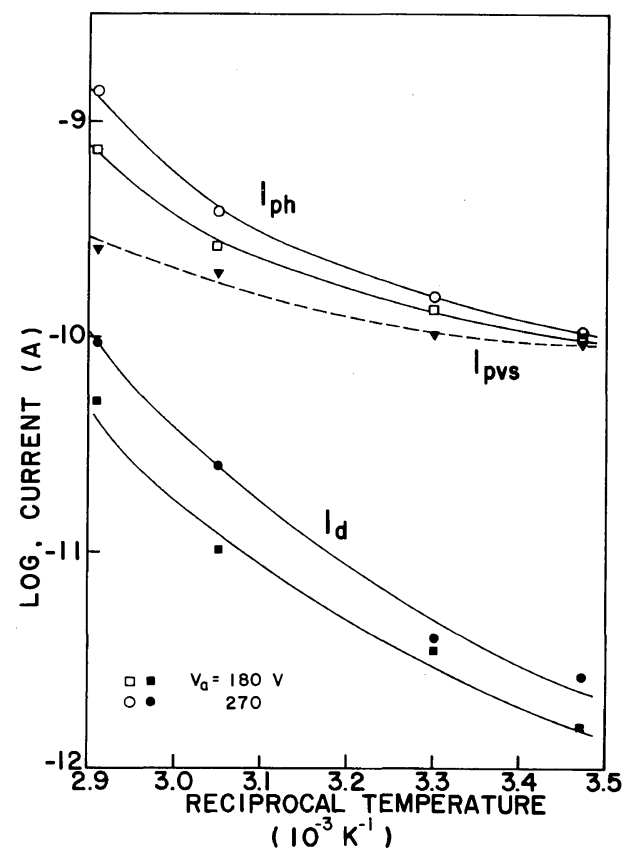

Figure 3. Arrhenius plots of the dark current $I_{\mathrm{d}}$, the photocurrent $I_{\mathrm{ph}}$ (steady state) and the photovoltaic current $I_{\text {pvs }}$ (steady state). For the $I_{\text {pvs }}$ measurement the applied voltage is absent $\left(V_{\mathrm{a}}=0\right)$.

partly by PVDF. Since the work function of $\mathrm{Au}$ is $4.58 \mathrm{eV}(\lambda=271 \mathrm{~nm})$, the direct photocarrier injection from a metal electrode is unexpectable. However, in general, polymers have fairly dense surface states (localized levels) with a wide distribution in energy depth and position and act as

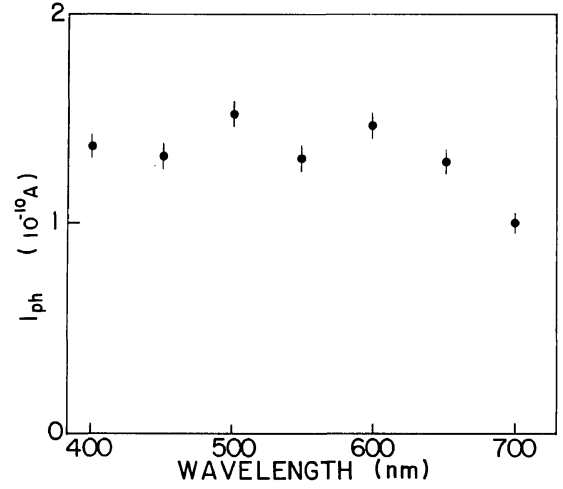

Figure 4. Action spectrum of the photocurrent $I_{\mathrm{ph}}$ in the unpoled sample $S_{\mathrm{A}}$.

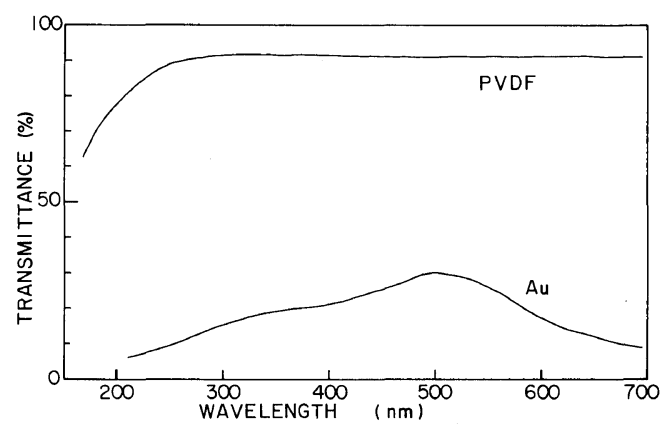

Figure 5. Optical transmittances of PVDF and the semitransparent $\mathrm{Au}$ electrode ( $c a .300 \AA$ thick).

carrier traps. In this case, trapped carriers are easily liberated by the low energy light of the wavelength studied here.

After doping the acrydine-orange dye into the original sample $\left(S_{\mathrm{A}}\right)$, the optical absorption edge of Sample $S_{\mathrm{B}}$ shifted towards a longer wavelength ( $c a$. $540 \mathrm{~nm}$ ). However, $I_{\mathrm{ph}}$ in $S_{\mathrm{B}}$ was slightly smaller than that in $S_{\mathrm{A}}$. This is similar to the result for photoconductive properties in dye-doped polyethylene naphthalates, ${ }^{9}$ where the dye molecules (dopants) behave as carrier traps rather than photocarrier sources. The photocurrent response in the poled sample $S_{\mathrm{C}}$ is quite different from that in $S_{\mathrm{A}}$; that is, $I_{\mathrm{ph}}$ shows an initial rise, followed by a gradual decrease due to the space charge formation and/or pyroelectric effect. This was confirmed by an inverse current spike observed at the instance of the light OFF [Figure 2(b)]. The applied field dependence of $I_{\mathrm{ph}}$ is also quite different for unpoled and poled samples, as shown in Figure 6. In the case of 


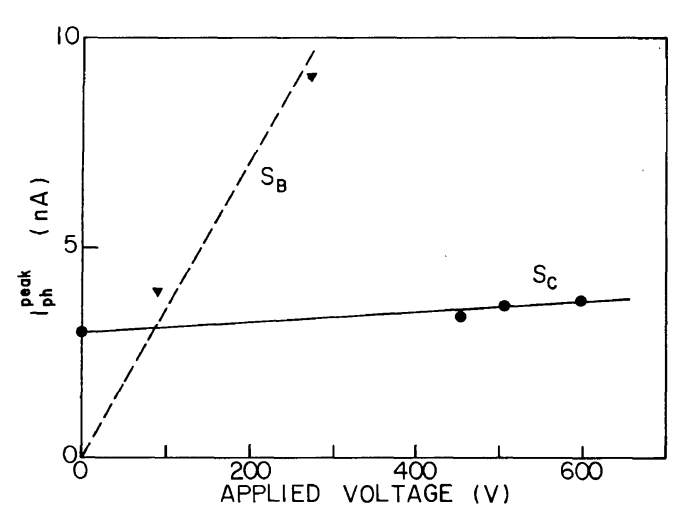

Figure 6. Applied field dependence of the photocurrent $I_{\mathrm{ph}}$ (peak value) in the unpoled sample $S_{\mathrm{B}}$ and the poled sample $S_{\mathrm{C}}$.

an unpoled sample, $I_{\mathrm{ph}}$ depends on the applied voltage $V_{\mathrm{a}}$ in a linear fashion, i.e., an ohmic behavior is observed. In contrast to this, in the case of a poled sample, $I_{\mathrm{ph}}$ changes linearly with $V_{\mathrm{a}}$, but is not zero at $V_{\mathrm{a}}=0$. This fact indicates the existence of a residual internal field in the poled sample.

\section{Internal Field in Poled PVDF}

A small current $I_{\mathrm{B}}$ is frequently observed across the sample, even when no applied field voltage $V_{\mathrm{a}}$ is present $\left(V_{\mathrm{a}}=0\right)$ and the temperature is kept constant. This behavior is more remarkable at higher temperatures, and has been observed in many other polymers such as poly(vinyl chloride) and poly(ethylene terephthalate). ${ }^{10}$ The current $I_{\mathrm{B}}$ is called a background current and may be due to a charges generated in the course of film preparation. In most cases, $I_{\mathrm{B}}$ can be made negligible by a field sweeping method, that is, by applying several times positive and negative high dc voltages alternatively to the sample at high temperature, or by illumination with intensive light. So is the case of samples $S_{\mathrm{A}}$ and $S_{\mathrm{B}}$, but sample $S_{\mathrm{C}}$ shows a very different behavior from this.

Figure 7 shows the applied voltage $\left(V_{\mathrm{a}}\right)$ dependence of a dark current $I_{\mathrm{d}}$ in $S_{\mathrm{C}}: I_{\mathrm{d}}$ depends on $V_{\mathrm{a}}$ in a linear fashion, and is nonzero at the zero field. Here, the positive $V_{\mathrm{a}}$ indicates an applied field parallel to the internal field caused by an arrangement of ferroelectric dipoles in the crystal. The residual field estimated from the intersect is $c a .40$ $\mathrm{V} / 30 \mu \mathrm{m}=0.013 \mathrm{MV} / \mathrm{cm}$. On the other hand, the internal electric field $E_{\mathrm{s}}$ caused by a spontaneous

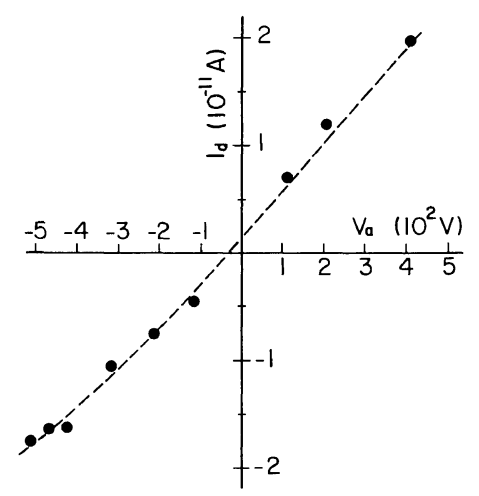

Figure 7. Changes in the dark current $I_{\mathrm{d}}$ with applied voltage in the poled sample $S_{\mathrm{C}}$.

polarization $P_{\mathrm{s}}$ of poled PVDF is given by

$$
E_{\mathrm{s}}=P_{\mathrm{s}} / \varepsilon
$$

where $\varepsilon$ is the dielectric constant. In the case of $S_{\mathrm{C}}$, $\varepsilon / \varepsilon_{0}=13$ and $P_{\mathrm{s}}=5 \mu \mathrm{C} / \mathrm{cm}^{2},{ }^{11}$ hence $E_{\mathrm{s}}=4.3$ $\mathrm{MV} / \mathrm{cm}$. The observed residual field is much smaller than $E_{\mathrm{s}}$ calculated from eq 1 . The reason for this is still not clear, but might possibly be the space charge effect or fluctuation in the $\mathrm{CF}_{2}$ dipoles of the $\beta$ form crystals, that is, only $1 \%$ spontaneous polarization is effective for the internal field formation. Whatever the mechanism may be, the internal field plays an important role in carrier transport in the PVDF film.

\section{Photovoltaic Effect}

The results described above suggest that electronic carriers are generated by light excitation, separated, and transported in the internal field. Hence, the photovoltaic effect in poled PVDF can be expected. Figure 8(a) indicates the photovoltaic current response in the poled PVDF sample $\left(S_{\mathrm{C}}\right)$ under steady state illumination. This is very similar to that obtained by Simhony and Shaulov ${ }^{12}$ for pyroelectric crystals. That is, the pyroelectric transient current consists of two relaxation processes characterized by thermal $\left(\tau_{\mathrm{T}}\right)$ and electrical $\left(\tau_{\mathrm{E}}\right)$ time constants, i.e.,

$$
I_{\text {pyro }}(t)=A\left\{\exp \left(-t / \tau_{\mathrm{T}}\right)-\exp \left(-t / \tau_{\mathrm{E}}\right)\right\},
$$

where $\tau_{\mathrm{E}}=\mathrm{RC}, \tau_{\mathrm{T}}=C_{\mathrm{T}} / G_{\mathrm{T}}, R$ and $C$ represent the resistance and the capacitance of the sample, $C_{\mathrm{T}}$ ( $=\rho C_{\mathrm{p}} v ; \rho$ the density, $C_{\mathrm{p}}$ the heat capacity and $v$ the volume) the thermal capacitance and $G_{\mathrm{T}}$ the 


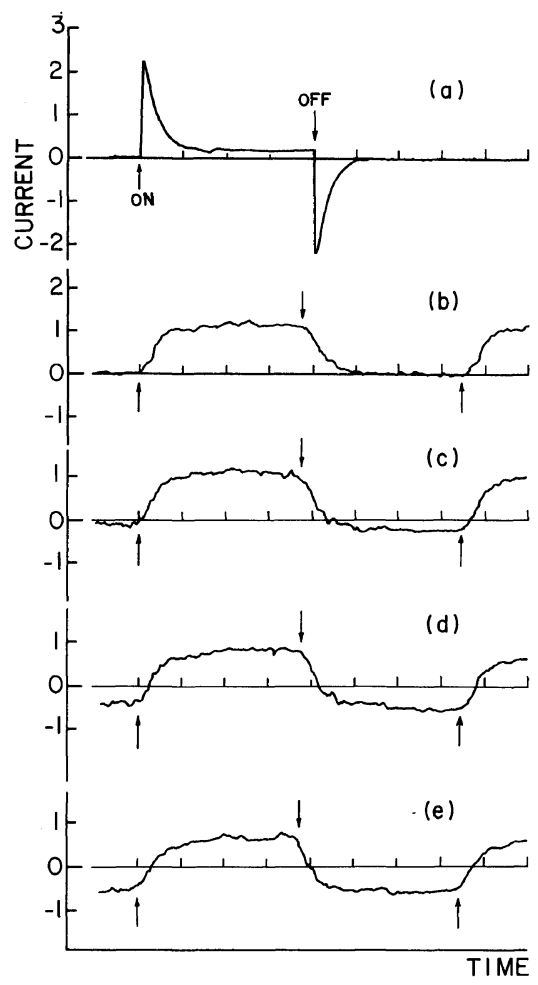

Figure 8. Time evolution of photovoltaic current $I_{\mathrm{pv}}$ in the poled sample $S_{\mathrm{C}}$ at a room temperature, (a) under the step-wise illumination ( $1 \mathrm{nA} / \mathrm{div} ; 5 \mathrm{~s} / \mathrm{div}$ ), and (b) - (e) under the pulsed illumination ( $2 \mathrm{nA} / \mathrm{div} ; 2 \mathrm{~ms} / \mathrm{div})$. (b) is for the 1st light pulse response, (c) 40 th pulse (ca. $0.5 \mathrm{~s}$ ), (d) 140th pulse (ca.2s), and (e) at the thermal equilibrium state (more than $20 \mathrm{~s}$ ).

thermal conductance of the coupling between the sample and its ambient. Das-Gupta and Doughty ${ }^{13}$ have analyzed the pyroelectric transient current in poled PVDF films in terms of eq 2 and obtained $\tau_{\mathrm{T}}=0.65 \mathrm{~s}$ and $\tau_{\mathrm{E}}=0.16 \mathrm{~s}$. In our case, $\tau_{\mathrm{T}}$ and $\tau_{\mathrm{E}}$ are estimated as $1.41 \mathrm{~s}$ and $0.11 \mathrm{~s}$, respectively. This discrepancy is reasonable because in our experiment the volume of the sample is larger by a factor of 3 and the thermal conductance under a vacuum of $10^{-5}$ Torr is smaller than in Das-Gupta et al.'s. The most remarkable difference between our results and theirs is the residual photovoltaic current $\left(I_{\mathrm{pvs}}\right)$ observed at the thermal equilibrium state (longer than $60 \mathrm{~s}$ ). As indicated by many investigators, ${ }^{13,14}$ a peak followed by a transient current may be related to the pyroelectricity, but the value of $I_{\mathrm{pvs}}$ is probably a real photovoltaic current.

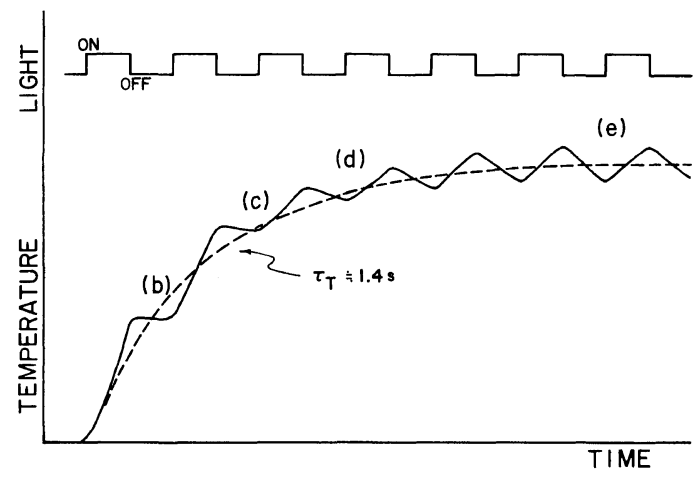

Figure 9. Schematic representation of the time evolution of temperature of the sample. For the dashed curve, see text.

Figure 8(b) shows the first pulse response of the photovoltaic current $I_{\mathrm{pv}} \cdot I_{\mathrm{pv}}$ increases sharply and reaches a steady state corresponding to $I_{\text {peak }}$ in Figure 8(a). Under repetitive illumination with light pulses, the response curve changes its form as shown in Figures 8(c)-(e). For the first pulse, $I_{\mathrm{pv}}$ vanishes rapidly when the light is OFF. After 40 pulses $(0.5 \mathrm{~s})$, the negative signal is observed by stopping the illumination due to the cooling of the sample [Figure 8(c)], as in the case of the result of Glass et al.'s. ${ }^{14}$ This behavior becomes more remarkable after 140 pulses (2s) [Figure 8(d)]. Finally, at the thermal equilibrium of the sample with its ambient, the response curve is nearly in the form of a square pulse [Figure $8(\mathrm{e})$ ]; that is, the sample is heated or cooled at a constant rate depending on whether the light is ON or OFF. Figure 9 presents a schematic representation of the time evolution of the temperature of the sample. The dashed curve indicates the average temperature change in the sample, and follows the relation,

$$
T(t)=T_{0}\left\{1-\exp \left(-t / \tau_{\mathrm{T}}\right)\right\}
$$

This result supports the view that the pyroelectric rather than the photovoltaic effect occurs in the poled PVDF film under photoexcitation. The initial response gives a response time of $0.4 \mathrm{~ms}$. This value is much smaller than the thermal relaxation time $\left(\tau_{\mathrm{T}}=1.41 \mathrm{~s}\right)$ estimated from Figure $8(\mathrm{a})$. The response time is a measure of the conversion rate of light energy into heat. The peak value of $I_{\mathrm{pv}}$ in Figure $8(\mathrm{a})\left(=I_{\text {peak }}\right)$ is plotted against exciting wavelength $(\lambda)$ in Figure 10. The $I_{\text {peak }}$ is almost 


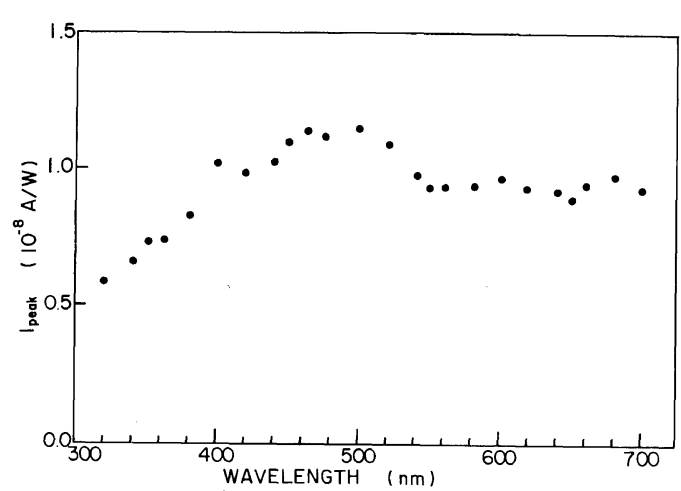

Figure 10. Action spectrum of the photovoltaic current $I_{\text {peak }}$ (the peak value) in the poled sample $S_{\mathrm{C}}$.

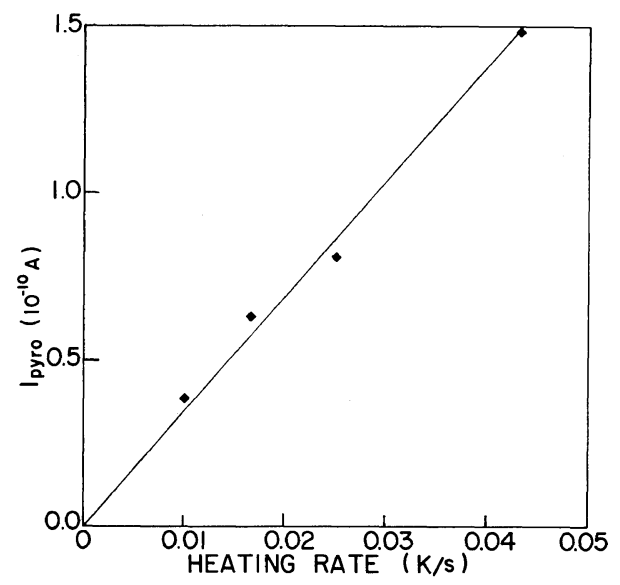

Figure 11. Relation between the pyroelectric current and the heating rate in the poled sample $S_{\mathrm{C}}$.

constant in a wavelength region exceeding $520 \mathrm{~nm}$, and then is followed by a small hump and a gradual decrease with decreasing $\lambda$. This gradual decrease may be attributed to light absorption by the deposited $\mathrm{Au}$ electrodes. This is also favorable for the pyroelectricity, since the change in $I_{\mathrm{ph}}$ with wavelength is negligible (Figure 4). Consequently, we investigated the difference between $I_{\text {pvs }}$ and the pyroelectricity due to the absorption of light in a poled PVDF sample.

As shown in Figure 11, the pyroelectric current changes linearly with the heating rate. The slope gives a pyroelectric coefficient $p$ of $4.4 \mathrm{nCK}^{-1}$ $\mathrm{cm}^{-2}$, which agrees with the literature value $\left(2.3 \mathrm{nC} \mathrm{K}^{-1} \mathrm{~cm}^{-2}\right) \cdot{ }^{13}$ Going on the assumption that all the light energy given to the sample changes into heat, the rate of temperature increase of the sample

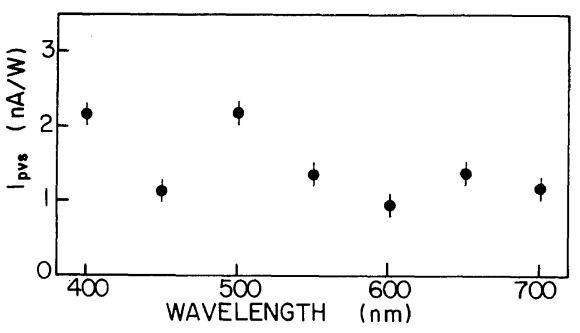

Figure 12. Action spectrum of the residual photovoltaic current $I_{\mathrm{pvs}}$ in the poled sample $S_{\mathrm{C}}$.

was estimated to be $c a .0 .085 \mathrm{~K} \mathrm{~s}^{-1}$. Here the light energy given to the sample, the absorption coefficient, the specific heat and the volume of the sample are $24.6 \mathrm{~mW}, 0.1,2.4 \mathrm{~J} \mathrm{~K}^{-1} \mathrm{~cm}^{-3}$ and $0.012 \mathrm{~cm}^{3}$, respectively. The calculated value of pyroelectric current $I_{\text {pyro }}^{\text {cal }} 0.38 \mathrm{nA} \mathrm{cm}^{-2}$, while the observed value under the same conditions $I_{\mathrm{pv}}^{\text {obs }}$ is $2 \pm 0.5 \mathrm{nA} \mathrm{cm}^{-2}$ (for the pulsed illumination). Therefore, $I_{\mathrm{pv}}^{\mathrm{obs}}$ is larger than $I_{\mathrm{pyro}}^{\mathrm{cal}}$ by a factor of 5 . If we take into consideration the light absorption by the deposited Au electrodes and the small thermal diffusivity of the polymer, the heating rate may be much larger than $0.085 \mathrm{~K} \mathrm{~s}^{-1}$ in the vicinity of the illuminated electrodes. $I_{\text {pyro }}^{\text {cal }}$ was underestimated in this sense. The reflection due to the Au electrode was also neglected, resulting in an overestimation of $I_{\text {pyro }}^{\text {cal }}$. Therefore, there is much ambiguity regarding $I_{\mathrm{pyro}}^{\mathrm{cal}}$, but it seems that $I_{\mathrm{pv}}^{\mathrm{obs}}$ is due not only to a pyroelectric effect but also to photocarrier generation and that the excess current corresponds to a photovoltaic current.

Figure 12 shows an action spectrum of the residual photovoltaic current $I_{\text {pvs }}$ in Sample $S_{\mathrm{C}}$. The bars indicate the uncertainty of the measurement. Hence $I_{\text {pvs }}$ is apparently independent of the exciting wavelength, and equal to $c a .1 .5 \mathrm{nA} \mathrm{W}^{-1}$. Thus, the short circuit photovoltaic current is quite small. Taking into account the fact that the dc conductance of $S_{\mathrm{C}}$ in the dark at room temperature is $4 \times 10^{-14}$ mho, we can estimate the open circuit photovoltaic $V_{\text {OC }}$ to be $4 \times 10^{4} \mathrm{~V}$. Therefore, the value of $V_{\text {OC }}$ is larger than the band gap voltage of polymers $\left(c a .9 \mathrm{eV}^{15}\right)$ by three decades. This fact supports the existence of an anomalous photovoltaic effect in the poled PVDF. The average value of $I_{\mathrm{pv}}^{\text {obs }}$ given in Figure 10 is about $10 \mathrm{nA} \mathrm{W}^{-1}$ for each exciting wavelength. Hence the conversion efficiency of light energy into the photovoltaic cur- 
rent $I_{\mathrm{pv}}$ was estimated to be $0.25 \%$. This conversion efficiency is quite low in comparison with amorphous Si solar cells ( $\mathrm{ca} .6 \%$ ), but the system used in this study is primitive; it is possible to make further improvements on the anti-reflection coating, enhancement of conductivity by reduction of the film thickness, and so on.

$I_{\mathrm{pvs}}$ increases with increasing temperature in the range of $15-80^{\circ} \mathrm{C}$ as shown in Figure 3 . The apparent activation energy calculated from the Arrhenius plot of $I_{\mathrm{pvs}}$ is somewhat temperature dependent but nearly equal to $0.15 \mathrm{eV}$ on the average. Above $80^{\circ} \mathrm{C}, I_{\text {pvs }}$ decreases with increasing temperature and can not be observed at about $110^{\circ} \mathrm{C}$. The reasons for this are primarily the background current $I_{\mathrm{B}}$ increases remarkably with temperature and covers the small $I_{\mathrm{pvs}}$ and secondly that since the piezoconstant $e_{33}$ also decreases with temperature in this temperature range, the internal field due to the spontaneous polarization of $\beta$ type crystal decreases.

\section{CONCLUSION}

We have studied the photoconductive and photovoltaic properties of poly(vinylidene fluoride) and confirmed the following points:

(1) In PVDF films electronic carriers can be generated by light illumination.

(2) In the case of poled PVDF, even when the external field is absent, the photogenerated carriers move along the internal field formed by the spontaneous polarization of $\beta$ crystals.

(3) The pyroelectric current is clearly observed in poled PVDF films by the photoexcitation, and additional current due to anomalous photovoltaic effect is also observed.

(4) The open circuit photovoltage was estimated as $4 \times 10^{4} \mathrm{~V}$, which is larger than the band gap potential of the polymer by three orders of magnitude.
(5) The doping of acrydine-orange dye into PVDF shifts the optical absorption edge, but negatively affects the carrier transport as traps.

Since the resistivity of PVDF is quite high, the photovoltaic power becomes small (the conversion efficiency $=c a .0 .25 \%$ ). In order to improve this disadvantage, one of the present authors (SM) developed a new technique for preparing an ultrathin film of PVDF ( $\beta$ form) by vacuum deposition. The details will be submitted in the near future.

\section{REFERENCES}

1. H. Naono, T. Gotoh, M. Matsumoto, S. Ibaraki, and Y. Rikow, 58th Convention of Audio Engineering Society, Preprint No. 1271 (1977) (in Japanese).

2. N. Chubachi and T. Sannomiya, Jpn. J. Appl. Phys., 16, 2259 (1977).

3. A. L. Robinson, Science, 200, 1371 (1978).

4. R. J. Phelan, Jr., R. J. Mahler, and A. R. Cook, Appl. Phys. Lett., 19, 337 (1971).

5. E. Fukada, Reports on Grant-in-Aid for Scientific Research, from the Ministry of Education, Science and Culture of Japan, 1981, p 68.

6. V. M. Fridkin, "Photoferroelectrics," Springer Verlag, Berlin-N.Y., 1979, Chapter 6.

7. A. M. Glass, D. von der Linde, and T. J. Negran, Appl. Phys. Lett., 25, 233 (1974).

8. T. Demura, K. Kamisako, and H. Sasabe, Rep. Prog. Polym. Phys. Jpn., 22, 361 (1979); ibid., 23, 437 (1980).

9. T. Nakayama, T. Demura, K. Kamisako, and H. Sasabe, Rep. Prog. Polym. Phys. Jpn., 23, 433 (1980).

10. H. Sasabe, Researches of Electrotechnical Laboratory, No. 721 (1971).

11. Kureha Chemical Industries, Technical Data for KF piezo-polymer.

12. M. Simhony and A. Shaulov, J. Appl. Phys., 42, 3741 (1971).

13. D. K. Das-Gupta and K. Doughty, J. Appl. Phys., 51, 1733 (1980).

14. A. M. Glass, J. H. McFee, and J. G. Bergman, Jr., J. Appl. Phys., 42, 5219 (1971).

15. K. J. Less and E. G. Wilson, J. Phys., C6, 3110 (1973). 